\title{
The graded structure induced by operators on a Hilbert space
}

\author{
By Kunyu Guo and Xudi Wang
}

(Received Apr. 29, 2016)

(Revised Nov. 5, 2016)

\begin{abstract}
In this paper we define a graded structure induced by operators on a Hilbert space. Then we introduce several concepts which are related to the graded structure and examine some of their basic properties. A theory concerning minimal property and unitary equivalence is then developed. It allows us to obtain a complete description of $\mathcal{V}^{*}\left(M_{z^{k}}\right)$ on any $H^{2}(\omega)$. It also helps us to find that a multiplication operator induced by a quasi-homogeneous polynomial must have a minimal reducing subspace. After a brief review of multiplication operator $M_{z+w}$ on $H^{2}(\omega, \delta)$, we prove that the Toeplitz operator $T_{z+\bar{w}}$ on $H^{2}\left(\mathbb{D}^{2}\right)$, the Hardy space over the bidisk, is irreducible.
\end{abstract}

\section{Introduction.}

In operator theory, it is of special interest to develop some algebraic methods to solve a variety of problems. There are the sophisticated classical theory of operator algebra and the fascinating K-theory. In general, there is a huge obstacle between algebra and topology, since the algebraic methods require many finiteness conditions, while the spirit of topology is based on the concept of continuousness. However, there are many structures that are not difficult to be handled. Once Douglas and Paulsen introduced the concept of Hilbert module [16], many researches on this new field are springing up $[\mathbf{1}],[\mathbf{2}],[\mathbf{8}],[\mathbf{9}],[\mathbf{1 4}],[\mathbf{1 5}],[\mathbf{1 9}],[\mathbf{2 1}],[\mathbf{2 2}],[\mathbf{2 3}]$. For a survey of Hilbert modules, one can see $[\mathbf{2 7}],[\mathbf{2 8}]$. Brown, Douglas and Fillmore also developed techniques of homological algebra to define an invariant called Ext, which is the cornerstone of the BDF theory $[4]$. On the other hand, the concept of Ext was also defined for Hilbert modules by Carlson and Clark [5], [6], [7]. Recently, there is a tendency that combines algebraic geometry and complex geometry with operator theory [11], [17]. As to commutative algebra, Hilbert-Samuel polynomials that are a class of Hilbert polynomials were already defined for Hilbert modules [18]. Intuitively, the graded structures of Hilbert spaces that Hilbert polynomials can be defined on should attract some attentions. Lately, motivated by [12], $[\mathbf{2 5}],[\mathbf{3 2}]$, we find that graded structure can be very useful in operator theory.

Let $L_{a}^{2}\left(\mathbb{D}^{2}\right)$ be the Bergman space over the bidisk $\mathbb{D}^{2}, M_{z+\alpha w}$ the multiplication operator induced by $z+\alpha w$ where $\alpha \neq 0$. Define $H_{n}=\operatorname{span}\left\{z^{k} w^{l}: k+l=n\right\}$ for each natural number $n$. Then, it is easy to see that

2010 Mathematics Subject Classification. Primary 47B37; Secondary 47C15, 47A80.

Key Words and Phrases. unilateral weighted shifts, reducing subspaces, multiplication operators, graded structure, Toeplitz operators.

This work was supported by NSFC(11371096). The second author also was supported by NSFC(11601418). 


$$
M_{z+\alpha w} H_{n} \subseteq H_{n+1} \text { and } M_{z+\alpha w}^{*} H_{n} \subseteq H_{n-1} .
$$

Together with $L_{a}^{2}\left(\mathbb{D}^{2}\right)=\bigoplus_{n=0}^{\infty} H_{n}$, this phenomenon leads us to an idea that we can handle this operator with the concept of graded structure. By using this idea, it is proved that $M_{z+\alpha w}$ is irreducible if and only if $|\alpha| \neq 1[\mathbf{1 2}],[\mathbf{3 2}]$. The same procedure is also effective for $H^{2}\left(\mathbb{D}^{2}\right)$, the Hardy space over the bidisk [25]. We will give the precise definition of graded structure in Section 3.

Once we define a graded structure for a Hilbert module, we ask whether we can characterize the minimal graded submodule, and how to define the isomorphism between two graded structures. We will distinguish two different cases for graded structures. The graded structure from the space $L_{a}^{2}\left(\mathbb{D}^{2}\right)$ and the operator $M_{z+\alpha w}$ is called truncated, since the $H_{n}$ is beginning at $n=0$. The other case is said to be non-truncated. Instead of minimality, we concentrate on the concept of stability, which is easier to be grasped than minimality. At last, we prove that an isomorphism between two truncated stable graded submodules is also graded in form.

Finally, we will give three important applications of this theory. First, we characterize the unitary equivalence between the reducing subspaces of $M_{z^{k}}$ which have been described in [31]. Second, we point out that a multiplication operator induced by a quasi-polynomial always has a minimal reducing subspace. Finally, we prove that on $H^{2}\left(\mathbb{D}^{2}\right)$, the Toeplitz operator induced by symbol $z+\bar{w}$ is irreducible.

\section{Preliminaries.}

\subsection{Review of module theory.}

To define a module structure on a Hilbert space, we start with the concept of module first. Let $\mathcal{A}$ be a ring, recall that a (left) $\mathcal{A}$-module $M$ is an abelian group with a ring homomorphism $\pi: \mathcal{A} \rightarrow \operatorname{End}(M)$, where $\operatorname{End}(M)$ is the endomorphism ring of the abelian group $M$. It follows that if we want to take $M$ a Hilbert space $H$, then it is natural that we should consider a ring homomorphism $\pi: \mathcal{A} \rightarrow \mathcal{B}(H)$, where $\mathcal{B}(H)$ is the bounded linear operator algebra on $H$. There are two things to consider. The first thing is that there are many topologies on $\mathcal{B}(H)$. We choose a topological $\operatorname{ring} \mathcal{A}$ to make that the ring homomorphism $\pi: \mathcal{A} \rightarrow(\mathcal{B}(H)$, SOT $)$ is continuous, where SOT means the strong operator topology. This is equivalent to say that the map $\mathcal{A} \times H \rightarrow H,(a, x) \mapsto$ $\pi(a) x$ is separately continuous. The second thing is that $\mathcal{B}(H)$ has an involution $*$. If $\mathcal{A}$ has also an involution $*$, then we require that $\pi$ is a $*$-homomorphism. Since $*$ may not be SOT-continuous, we further require that $\pi(\mathcal{A})$ is an SOT-closed $*$-subalgebra of $\mathcal{B}(H)$. However, the action of $\mathcal{A}$ on $H$ is the same as $\pi(\mathcal{A})$ on $H$ as long as the structure of $H$ is concerned. In this paper, it will suffice that we restrict our attention to the case that $\mathcal{A}$ is an SOT-closed subalgebra (or $*$-subalgebra) of $\mathcal{B}(H)$. Then we shall say that $H$ is an $\mathcal{A}$-module.

A submodule $M$ of an $\mathcal{A}$-module $H$ is defined by a closed subspace of $H$ which is closed under module operators. This is equivalent to say that $M$ is an $\mathcal{A}$-invariant subspace of $H$. Therefore, there is a one-to-one correspondence between submodules of $H$ and $\mathcal{A}$-invariant subspaces of $H$. Moreover, if $\mathcal{A}$ is a $*$-subalgebra, then it is equivalent to say that $M$ is an $\mathcal{A}$-reducing subspace of $H$. In this case there is a one-to- 
one correspondence between submodules of $H$ and $\mathcal{A}$-reducing subspaces of $H$.

We now turn to the graded module theory. In commutative algebra, a graded ring $A$ is a commutative ring together with a direct sum decomposition

$$
A=\bigoplus_{n=-\infty}^{+\infty} A_{n}
$$

as abelian groups, such that

$$
A_{n} A_{m} \subseteq A_{n+m} \text { for all } n, m .
$$

Then, a graded $A$-module $M$ is an $A$-module with decomposition

$$
M=\bigoplus_{n=-\infty}^{+\infty} M_{n}
$$

as abelian groups, such that

$$
A_{n} M_{m} \subseteq M_{n+m} \text { for all } n, m \text {. }
$$

We want to establish a graded structure on an $\mathcal{A}$-module $H$ in this paper. Although $\mathcal{A}$ need not be commutative, this is not an essential obstacle, since we mainly concern submodules of $H$. The formal definitions of these are given in Section 3.

\section{2. $\mathcal{S}$-calculus.}

Let $H$ be a Hilbert space and $T \in \mathcal{B}(H)$. Put $\mathcal{W}^{*}(T)$ the von Neumann algebra generated by $T$, and $\mathcal{V}^{*}(T)=\mathcal{W}^{*}(T)^{\prime}$. We know that there is a one-to-one correspondence between reducing subspaces of $T$ and projections in $\mathcal{V}^{*}(T)$. In this paper, we mainly study the $\mathcal{W}^{*}(T)$-module $H$. It is not hard to see that there is also a one-to-one correspondence between reducing subspaces of $T$ and submodules of $H$.

Throughout the paper, we denote by $\mathbb{Z}$ the set of integers, $\mathbb{Z}_{+}$the set of non-negative integers, and $\mathbb{N}$ the set of positive integers. Let us divide $\mathcal{W}^{*}(T)$ into many parts. For each $n \in \mathbb{Z}$, we let

$$
\mathcal{S}^{n}=\left\{\prod_{k=1}^{N} T^{* j_{k}} T^{i_{k}}, N \in \mathbb{N}, i_{k}, j_{k} \in \mathbb{Z}_{+}: \sum_{k=1}^{N}\left(i_{k}-j_{k}\right)=n\right\} .
$$

Note, $I \in \mathcal{S}^{0}$.

We have the following key lemma.

Lemma 2.1. Let $n \geq 0$ and $A=\prod_{k=1}^{N} T^{* j_{k}} T^{i_{k}}, N \in \mathbb{N}, i_{k}, j_{k} \in \mathbb{Z}_{+}$,

$$
\sum_{k=1}^{N}\left(i_{k}-j_{k}\right)=n
$$

Then for any integer $m$ with $0 \leq m \leq n$, there exists some $B \in \mathcal{S}^{m}$ and $C \in \mathcal{S}^{n-m}$ such that $A=B C$. 
Proof. Note that the lemma is true when $n=0, m=0$ or $m=n$. For example, if $m=0$, then take $B=I, C=A$. Next we use induction on $N$. If $N=1$, i.e. $A=T^{* j} T^{i}$. In this case $i-j=n$, we can put $B=T^{* j} T^{i-n+m}, C=T^{n-m}$.

Now assume that the lemma is true for $N=r$ and consider the case $N=r+1$,

$$
A=\left(\prod_{k=1}^{r} T^{* j_{k}} T^{i_{k}}\right)\left(T^{* s} T^{t}\right), \quad \sum_{k=1}^{r}\left(i_{k}-j_{k}\right)+(t-s)=n \geq 0 .
$$

Set $\sigma=\sum_{k=1}^{r}\left(i_{k}-j_{k}\right)$. If $\sigma \geq m$, by assumption, there exist $B \in \mathcal{S}^{m}, C^{\prime} \in \mathcal{S}^{\sigma-m}$ such that $\prod_{k=1}^{r} T^{* j_{k}} T^{i_{k}}=B C^{\prime}$. Put $C=C^{\prime} T^{* s} T^{t}$, then $C \in \mathcal{S}^{n-m}$ and $A=B C$. If $\sigma<m$, Put $B=\left(\prod_{k=1}^{r} T^{* j_{k}} T^{i_{k}}\right)\left(T^{* s} T^{t-n+m}\right) \in \mathcal{S}^{m}, C=T^{n-m} \in \mathcal{S}^{n-m}$, then $A=B C$.

This completes the lemma by induction.

Before the proposition for $\mathcal{S}^{n}$ is given below, we introduce some notations. For any $F \subseteq H$ and $\mathcal{A} \subset \mathcal{B}(H)$, denote by $[F]$ the closed subspace spanned by $F$. Define

$$
\mathcal{A} F=[\{A f: A \in \mathcal{A}, f \in F\}] .
$$

If $F=\{f\}$, then write $\mathcal{A} f=\mathcal{A} F$. Note that if $T \in \mathcal{B}(H)$, then $T F \subseteq\{T\} F$, and the equality holds if and only if $T F$ is a closed subspace.

Proposition 2.2. We have the following

(a) $\mathcal{S}^{0}$ is a monoid, i.e., it is closed under the operator product and $I$ is its identity.

(b) $\left(\mathcal{S}^{n}\right)^{*}=\mathcal{S}^{-n}, n \in \mathbb{Z}$.

(c) $\mathcal{S}^{n} \mathcal{S}^{m} \subseteq \mathcal{S}^{m+n}, n, m \in \mathbb{Z}$.

(d) If $n m \geq 0$, then $\mathcal{S}^{n} \mathcal{S}^{m}=\mathcal{S}^{n+m}$.

(e) If $F \subseteq H$, then $\mathcal{S}^{n}\left(\mathcal{S}^{m} F\right)=\left(\mathcal{S}^{n} \mathcal{S}^{m}\right) F, n, m \in \mathbb{Z}$.

Proof. (a), (b), (c) is trivial.

If both $n$ and $m$ are non-negative, then $\mathcal{S}^{n} \mathcal{S}^{m}=\mathcal{S}^{n+m}$ by (c) and Lemma 2.1. Otherwise, it follows from (a) that

$$
\mathcal{S}^{n} \mathcal{S}^{m}=\left(\mathcal{S}^{-n}\right)^{*}\left(\mathcal{S}^{-m}\right)^{*}=\left(\mathcal{S}^{-m} \mathcal{S}^{-n}\right)^{*}=\left(\mathcal{S}^{-n-m}\right)^{*}=\mathcal{S}^{n+m} .
$$

Hence (d) is true.

(e) is the consequence of the SOT-continuousness of left multiplication by an operator.

Now, for any operator $T \in \mathcal{B}(H)$, we can produce the set $\mathcal{S}^{n}$ for any integer $n$. Henceforth, we will call this procedure the $\mathcal{S}$-calculus for $T$.

We state here a result about a sufficient condition for a reducing subspace to be minimal that is given by $\mathcal{S}$-calculus. 
Proposition 2.3. Suppose that $H$ is a Hilbert space, $T \in \mathcal{B}(H)$, and $\mathcal{S}$-calculus for $T$ satisfies $\bigcap_{n \in \mathbb{Z}_{+}} \mathcal{S}^{n} H=0$. Let $M$ be a reducing subspace of $T$. If $\operatorname{dim} M \ominus \mathcal{S}^{1} M=1$, then $M$ is minimal.

Proof. First, we claim that for any non-zero subspace $K, \mathcal{S}^{1} K \neq K$. Otherwise, for any $n>0$, we have $\mathcal{S}^{n} K=K$. It follows from $\mathcal{S}^{0} K \supseteq K$ that

$$
K=\bigcap_{n \in \mathbb{Z}_{+}} \mathcal{S}^{n} K \subseteq \bigcap_{n \in \mathbb{Z}_{+}} \mathcal{S}^{n} H=\{0\} .
$$

This contradicts the fact that $K \neq 0$.

Now suppose that $M$ is a reducing subspace of $T$ satisfying $\operatorname{dim} M \ominus \mathcal{S}^{1} M=1$, then $\mathcal{S}^{1} M \subseteq M$. If $M=M_{1} \oplus M_{2}$, where $M_{1}$ and $M_{2}$ are two non-zero reducing subspace of $T$. According to the claim above, $\operatorname{dim} M_{i} \ominus \mathcal{S}^{1} M_{i} \geq 1, i=1,2$. However,

$$
\operatorname{dim} M \ominus \mathcal{S}^{1} M=\operatorname{dim} M_{1} \ominus \mathcal{S}^{1} M_{1}+\operatorname{dim} M_{2} \ominus \mathcal{S}^{1} M_{2} \geq 2,
$$

this is a contradiction. Thus $M$ must be minimal.

\subsection{Weighted sequence spaces.}

Let $\omega=\left\{\omega_{0}, \omega_{1}, \cdots, \omega_{n}, \cdots\right\}$ be a sequence of positive numbers. We consider the space of sequences $f=\{\hat{f}(n)\}$ such that

$$
\|f\|^{2}=\|f\|_{\omega}^{2}=\sum_{n=0}^{+\infty}|\hat{f}(n)|^{2} \omega_{n}<\infty .
$$

Let $f(z)=\sum_{n=0}^{+\infty} \hat{f}(n) z^{n}$ be the formal power series in $z$. Then we shall denote the space $H^{2}(\omega)$. It is clear that $H^{2}(\omega)$ is a Hilbert space with the inner product

$$
\langle f, g\rangle=\sum_{n=0}^{+\infty} \hat{f}(n) \overline{\hat{g}(n)} \omega_{n} .
$$

It is easy to see that $\left\{z^{n} / \sqrt{\omega_{n}}\right\}_{n \in \mathbb{Z}_{+}}$is an orthonormal basis for $H^{2}(\omega)$. The proposition below shows that $H^{2}(\omega)$ can become a analytic function space over an open disc.

Proposition $2.4([\mathbf{3 0}])$. Let $r_{2}(\omega)=\liminf _{n \rightarrow \infty} \omega(n)^{1 / 2 n}$, then the disc

$$
\Delta_{2}(\omega)=\left\{z:|z|<r_{2}(\omega)\right\}
$$

is the largest open disc in which all the power series in $H^{2}(\omega)$ converge.

Now consider the linear operator $M_{p}$ of multiplication by a polynomial $p$ on $H^{2}(\omega)$ :

$$
\left(M_{p} f\right)(z)=f(z) p(z) .
$$

It is well known that $M_{z}$ is bounded on $H^{2}(\omega)$ if and only if $\sup _{n}\left(\omega_{n+1}\right) / \omega_{n}<\infty$. In this case $M_{p}$ is bounded and $M_{p}=p\left(M_{z}\right)$. Throughout the paper we assume that $M_{z}$ 
is always bounded. If we let $\alpha_{n}=\sqrt{\left(\omega_{n+1}\right) / \omega_{n}}$, then $M_{z}$ is unitarily equivalent to the unilateral weighted shift with weight sequence $\alpha$.

We can use these to construct some spaces of analytic functions of several variables. For two variables, let $\delta$ be another sequence of positive numbers satisfying $\sup _{n}\left(\delta_{n+1}\right) / \delta_{n}<\infty$, we define $H^{2}(\omega, \delta)=H^{2}(\omega) \otimes H^{2}(\delta)$ as the Hilbert space consisting of analytic functions

$$
f(z, w)=\sum_{n, m=0}^{+\infty} a_{n, m} z^{n} w^{m}
$$

in the bidisk $\Delta_{2}(\omega) \times \Delta_{2}(\delta)$ such that

$$
\|f\|^{2}=\|f\|_{\omega, \delta}^{2}=\sum_{n, m=0}^{+\infty} \omega_{n} \delta_{m}\left|a_{n, m}\right|^{2}<\infty .
$$

Then it is clear that $M_{p+q}$ on $H^{2}(\omega, \delta)$ can be written as $M_{p} \otimes I+I \otimes M_{q}$ if $p$ is a polynomial of $z$ and $q$ is a polynomial of $w$, where $M_{p}$ is on $H^{2}(\omega)$ and $M_{q}$ is on $H^{2}(\delta)$.

In Section 4, we will give a complete description of $\mathcal{V}^{*}\left(M_{z^{k}}\right)$ on any $H^{2}(\omega)$.

\section{Graded structure.}

3.1. Some definitions and examples.

Let $H$ be a Hilbert space and $\mathcal{A} \subseteq \mathcal{B}(H)$ be an SOT-closed subalgebra. Notice that $H$ is a left $\mathcal{A}$-module. We begin the study of graded structures as follows.

Definition 3.1. We say that $\mathcal{A}$ is a graded $\operatorname{ring}$ and $H$ is a graded $\mathcal{A}$-module, if we have

$$
\mathcal{A}={\overline{\sum_{n=-\infty}^{+\infty} \mathcal{A}_{n}}}^{\mathrm{SOT}}
$$

with $\mathcal{A}_{n} \mathcal{A}_{m} \subseteq \mathcal{A}_{n+m}, n, m \in \mathbb{Z}$, where

$$
\sum_{n=-\infty}^{+\infty} \mathcal{A}_{n}=\left\{\sum_{n=-\infty}^{+\infty} a_{n}: a_{n} \in \mathcal{A}_{n}, a_{n} \neq 0 \text { for finitely many } n\right\}
$$

and

$$
H=\bigoplus_{n=-\infty}^{+\infty} H_{n}
$$

here all $H_{n}$ are closed subspaces of $H$, satisfying the following condition

$$
\mathcal{A}_{n} H_{m} \subseteq H_{n+m}, \quad n, m \in \mathbb{Z} .
$$

The members of $\mathcal{A}_{n}$ or $H_{n}$ are called homogeneous elements of degree $n$. (1) is called a homogeneous decomposition of $H$. 
From now on, when we say that $H$ is a graded $\mathcal{A}$-module, it means that $H$ has a homogeneous decomposition (1). Generally, there are two cases:

(a) There exists a sequence of integers $\left\{n_{k}\right\}$ such that $H_{n_{k}} \neq 0$ and $n_{k} \rightarrow-\infty$;

(b) There exists an integer $n_{0}$ such that $H_{n}=0$ for all integers $n<n_{0}$ and $H_{n_{0}} \neq 0$.

If we define

$$
n(H)=\inf \left\{n: H_{n} \neq 0\right\}
$$

then for the case (a), $n(H)=-\infty$ and for the case (b), $n(H)=n_{0} \in \mathbb{Z}$. Let's point out that $n(H)$ is dependent on the specific decomposition (1). We shall say that $H$ is truncated if $n(H) \in \mathbb{Z}$. When $H$ is truncated, we may rewrite (1) as follows

$$
H=\bigoplus_{n=n_{0}}^{+\infty} H_{n}
$$

Next, we study graded submodule.

Definition 3.2. We say that a submodule $M$ of the graded $\mathcal{A}$-module $H$ is graded, if $M$ has the following decomposition

$$
M=\bigoplus_{n=-\infty}^{+\infty} M_{n}
$$

where $M_{n}$ is a closed subspace of $H_{n}$ for each $n$.

We have the following propositions whose proofs are easy.

Proposition 3.3. Let $H$ be a graded $\mathcal{A}$-module and let $M$ be a graded submodule of $H$. Then $\mathcal{A}_{n} M_{m} \subseteq M_{n+m}, n, m \in \mathbb{Z}$. Furthermore, we have

$$
M_{n}=M \cap H_{n}, \quad n \in \mathbb{Z} .
$$

Conversely, if

$$
M=\bigoplus_{n=-\infty}^{+\infty} M \cap H_{n},
$$

then $M$ is a graded submodule of $H$.

Proposition 3.4. Let $H$ be a graded $\mathcal{A}$-module and let $M$ be a graded submodule of $H$. Then for each $m \in \mathbb{Z}$, we have

$$
\mathcal{A}_{m} M=\bigoplus_{n=-\infty}^{+\infty} \mathcal{A}_{m} M_{n}
$$

In this paper, we restrict our attention to graded modules and their graded submodules. A few examples of graded rings will be given as follows. 
EXAMPLE 3.5. Let $\left\{T_{1}, \cdots, T_{d}\right\}$ be a collection of commuting operators acting on a Hilbert space $H, \mathbb{C}\left[T_{1}, \cdots, T_{d}\right]$ be the space consisting of polynomials generated by $\left\{T_{1}, \cdots, T_{d}\right\}$, and $\mathcal{A}=\overline{\mathbb{C}\left[T_{1}, \cdots, T_{d}\right]}{ }^{\mathrm{SOT}}$. Set

$$
\mathcal{A}_{n}=\left\{p\left(T_{1}, \cdots, T_{d}\right): p \text { is a homogeneous polynomial of degree } n\right\}
$$

and $\mathcal{A}_{n}=0$ when $n<0$. In this case,

$$
\mathcal{A}=\overline{\sum_{n=-\infty}^{+\infty} \mathcal{A}_{n}}
$$

and $\mathcal{A}_{n} \mathcal{A}_{m} \subseteq \mathcal{A}_{n+m}, n, m \in \mathbb{Z}$.

Example 3.6. For any $T \in \mathcal{B}(H)$, we apply the $\mathcal{S}$-calculus for $T$. Put $\mathcal{A}=\mathcal{W}^{*}(T)$, and $\mathcal{A}_{n}=\operatorname{span} \mathcal{S}^{n}$. It is easy to show that $\mathcal{A}_{n} \mathcal{A}_{m} \subseteq \mathcal{A}_{n+m}$ by Proposition $2.2, n, m \in \mathbb{Z}$. Then,

$$
\mathcal{A}={\overline{\sum_{n=-\infty}^{+\infty} \mathcal{A}_{n}}}^{\mathrm{SOT}}
$$

In this time, we call such graded $\mathcal{A}$-module be a graded $\mathcal{S}$-module. Note that for any $F \subseteq H$ and $n \in \mathbb{Z}$, we have $\mathcal{A}_{n} F=\mathcal{S}^{n} F$.

A few examples of graded modules will be given as follows. In each example, we can verify that

$$
H=\bigoplus_{n=-\infty}^{+\infty} H_{n}
$$

and

$$
\mathcal{S}^{n} H_{m} \subseteq H_{n+m}, \quad n, m \in \mathbb{Z} .
$$

So they are all graded $\mathcal{S}$-modules.

Example 3.7. Let $H=H^{2}(\omega), T=M_{z}$. Put $H_{n}=\mathbb{C} z^{n}$ if $n \geq 0$, and $H_{n}=0$ if $n<0$.

Example 3.8. Let $H=H^{2}(\omega, \delta), T=M_{z+w}$. Put $H_{n}=\operatorname{span}\left\{z^{i} w^{j}: i+j=n\right\}$ if $n \geq 0$, and $H_{n}=0$ if $n<0$.

The above examples are all truncated. We mainly concern such type in the paper. Next we give a non-truncated example, which is related to the operator $M_{z}+M_{w}^{*}$ on $H^{2}(\omega, \delta)$.

Example 3.9. Let $H=H^{2}(\omega, \delta), T=M_{z}+M_{w}^{*}$. Put $H_{n}=\left[\left\{z^{i} w^{j}: i-j=n\right\}\right]$.

It is easy to check that if a set $E$ is consisting of homogeneous elements of same degree, then a submodule generated by $E$ is graded again, and it has the following form. 
Proposition 3.10. Let $H$ be a graded $\mathcal{A}$-module. If $E \subseteq H_{k}$ for some $k$, then the submodule $[E]_{\mathcal{A}}$ generated by $E$ is graded with the following homogeneous decomposition

$$
[E]_{\mathcal{A}}=\bigoplus_{n=-\infty}^{+\infty} \mathcal{A}_{n} E
$$

Now, we will introduce a new important concept. Recall that we have already defined the $n(H)$ for a graded $\mathcal{A}$-module $H$.

Definition 3.11. Let $H$ be a graded $\mathcal{A}$-module and $n_{0}=n(H)$. Then $H$ is said to be stable if $\mathcal{A}_{n} H_{m}=H_{n+m}$ when $m \geq n_{0}$ and $n \geq 0$. In this case, (1) is called a stable decomposition of $H$.

There is an interesting connection between stable and truncated that will be shown in the following proposition.

Proposition 3.12. If $H$ is a stable graded $\mathcal{A}$-module, then $H$ has a truncated stable decomposition if and only if $H \ominus \mathcal{A}_{1} H \neq 0$. In this case, the H's stable decomposition is uniquely determined.

Proof. If $H$ has a truncated stable decomposition as follows

$$
H=\bigoplus_{n=n_{0}}^{+\infty} H_{n}
$$

where $H_{n_{0}} \neq 0$. Then by stability we have $\mathcal{A}_{1} H_{n}=H_{n+1}$ when $n \geq n_{0}$. Thus

$$
\mathcal{A}_{1} H=\bigoplus_{n=n_{0}+1}^{+\infty} H_{n}
$$

hence

$$
H \ominus \mathcal{A}_{1} H=H_{n_{0}} \neq 0
$$

Notice that if $n \geq 0$, then

$$
H_{n_{0}+n}=\mathcal{A}_{n} H \ominus \mathcal{A}_{n+1} H
$$

We can conclude that the truncated stable decomposition, if it exists, is uniquely determined.

If any stable decomposition of $H$ is not truncated, then a stable decomposition

$$
H=\bigoplus_{n=-\infty}^{+\infty} H_{n}
$$

has the property that $n(H)=-\infty$. In this case, we have $\mathcal{A}_{1} H_{n-1}=H_{n}$ for any $n \in \mathbb{Z}$. It yields to $H=\mathcal{A}_{1} H$, i.e. $H \ominus \mathcal{A}_{1} H=0$. 
In summary, it is proved that $H$ has a truncated stable decomposition if and only if $H \ominus \mathcal{A}_{1} H \neq 0$.

At last, if $H \ominus \mathcal{A}_{1} H \neq 0$, then any stable decomposition of $H$ must be truncated, hence the stable decompostion of $H$ is uniquely determined.

Inspired by Proposition 2.3, we introduce the following concept.

Definition 3.13. We say that a graded $\mathcal{A}$-module $H$ is $\mathcal{A}$-regular, if it satisfies the following

$$
\bigcap_{n \in \mathbb{Z}_{+}} \mathcal{A}_{n} H=0 .
$$

It is easy to see that if $H$ is $\mathcal{A}$-regular, then all of its submodules are also $\mathcal{A}$-regular. Let's point out that $\mathcal{A}$-regularity can be guaranteed by truncatedness.

Proposition 3.14. A truncated graded $\mathcal{A}$-module is $\mathcal{A}$-regular.

Proof. Suppose that $H$ is a truncated graded $\mathcal{A}$-module, then there is a decomposition as follows

$$
H=\bigoplus_{n=n_{0}}^{+\infty} H_{n}
$$

For any $m \geq 0$ we have

$$
\mathcal{A}_{m} H \subseteq \bigoplus_{n=n_{0}+m}^{+\infty} H_{n}
$$

It follows that

$$
\bigcap_{m \in \mathbb{Z}_{+}} \mathcal{A}_{m} H=0
$$

Thus $H$ is $\mathcal{A}$-regular.

We conclude this subsection with a brief discussion of minimal graded submodules.

Definition 3.15. We say that a non-zero graded submodule $M$ of a graded $\mathcal{A}$ module $H$ is $\mathcal{A}$-minimal, if $M$ is minimal in the set of all non-zero graded submodules of $H$. Furthermore, if $H$ is its $\mathcal{A}$-minimal graded submodule, then $H$ is called $\mathcal{A}$-irreducible.

Thus, an $\mathcal{A}$-minimal graded submodule need not be a minimal submodule, and a minimal submodule which is also graded is an $\mathcal{A}$-minimal graded submodule.

\subsection{Some results on graded $\mathcal{S}$-modules.}

In this subsection, we mainly study non-zero graded $\mathcal{S}$-modules. Suppose $T \in \mathcal{B}(H)$ and $\mathcal{S}$-calculus for $T$ is applied such that $H$ is a graded $\mathcal{S}$-module. 
Recall that an $\mathcal{S}$-minimal graded submodule of a graded $\mathcal{S}$-module $H$ is a minimal element in the set of all non-zero graded submodules of $H$. The next proposition points out that all $\mathcal{S}$-minimal graded submodules are stable submodules.

Proposition 3.16. $\quad$ Every $\mathcal{S}$-minimal graded submodule of a graded $\mathcal{S}$-module is stable.

Proof. Suppose that $H$ is a graded $\mathcal{S}$-module, and $M$ is an $\mathcal{S}$-minimal graded submodule of $H$. If $M=\bigoplus_{n=-\infty}^{+\infty} M_{n}$ is a homogeneous decomposition of $M$, then by minimal property of $M$, we have $\left[M_{m}\right]_{\mathcal{S}}=M$, where $m \in \mathbb{Z}, M_{m} \neq 0$. Denote by $n_{0}=n(M)$. We divide into two cases.

(a) $n_{0} \neq-\infty$, so $M_{n_{0}} \neq 0$. By Proposition 3.10 , we get

$$
\left[M_{n_{0}}\right]_{\mathcal{S}}=\bigoplus_{n=0}^{+\infty} \mathcal{S}^{n} M_{n_{0}}
$$

As $\left[M_{n_{0}}\right]_{\mathcal{S}}=M$, for all $n \geq 0$ we have $\mathcal{S}^{n} M_{n_{0}}=M_{n+n_{0}}$. If $m \geq n_{0}$ and $n \geq 0$, then

$$
\mathcal{S}^{n} M_{m}=\mathcal{S}^{n} M_{m-n_{0}+n_{0}}=\mathcal{S}^{n} \mathcal{S}^{m-n_{0}} M_{n_{0}}=\mathcal{S}^{n+m-n_{0}} M_{n_{0}}=M_{n+m},
$$

hence $M$ is stable.

(b) $n_{0}=-\infty$. That is to say, for any $m$, there exists a $k \leq m$ such that $M_{k} \neq 0$. By Proposition 3.10, we get

$$
\left[M_{k}\right]_{\mathcal{S}}=\bigoplus_{n=-\infty}^{+\infty} \mathcal{S}^{n} M_{k}
$$

Since $\left[M_{k}\right]_{\mathcal{S}}=M$, we know $\mathcal{S}^{n} M_{k}=M_{n+k}$ for all $n$. If $n \geq 0$, then

$$
\mathcal{S}^{n} M_{m}=\mathcal{S}^{n} M_{m-k+k}=\mathcal{S}^{n} \mathcal{S}^{m-k} M_{k}=\mathcal{S}^{n+m-k} M_{k}=M_{n+m} .
$$

Since $m$ was arbitrary, $M$ is stable.

Next we will look for an $\mathcal{S}$-minimal graded submodule in stable graded submodules. It follows from Propositions 3.12 and 3.16 that the truncated graded decomposition of a truncated $\mathcal{S}$-minimal graded submodule must be stable and be uniquely determined.

We already proved that all $\mathcal{S}$-minimal graded submodules are stable. Then we consider when a stable submodule is $\mathcal{S}$-minimal.

Proposition 3.17. If $H$ is a graded $\mathcal{S}$-module, $E \subseteq H_{k}$, and $E$ satisfies the following

(a) There is an $n_{0}$ such that if $n<n_{0}$ then $\mathcal{S}^{n} E=0$;

(b) If $n \geq n_{0}$, then $\mathcal{S}^{1}\left(\mathcal{S}^{n} E\right)=\mathcal{S}^{n+1} E$;

(c) $\operatorname{dim} \mathcal{S}^{n_{0}} E=1$.

Then the submodule $[E]_{\mathcal{S}}$ generated by $E$ is a minimal $\mathcal{S}$-submodule, hence is $\mathcal{S}$-minimal. 
Proof. According to proposition 3.10,

$$
[E]_{\mathcal{S}}=\bigoplus_{n=-\infty}^{+\infty} \mathcal{S}^{n} E
$$

By condition (a),

$$
[E]_{\mathcal{S}}=\bigoplus_{n=n_{0}}^{+\infty} \mathcal{S}^{n} E
$$

By condition (b),

$$
[E]_{\mathcal{S}} \ominus \mathcal{S}^{1}[E]_{\mathcal{S}}=\left(\bigoplus_{n=n_{0}}^{+\infty} \mathcal{S}^{n} E\right) \ominus\left(\bigoplus_{n=n_{0}+1}^{+\infty} \mathcal{S}^{n} E\right)=\mathcal{S}^{n_{0}} E
$$

By conditon (c),

$$
\operatorname{dim}[E]_{\mathcal{S}} \ominus \mathcal{S}^{1}[E]_{\mathcal{S}}=1 .
$$

Since $[E]_{\mathcal{S}}$ is surely truncated, $[E]_{\mathcal{S}}$ is $\mathcal{S}$-regular by Proposition 3.14 . According to Proposition 2.3, $[E]_{\mathcal{S}}$ is minimal.

Finally, we will discuss the isomorphism problem. Suppose that $M$ (resp. $N$ ) is an $\mathcal{S}$-module, and the $\mathcal{S}$-calculus is given by $A$ (resp. $B$ ). $M$ and $N$ are said to be $\mathcal{S}$-isomorphic, if there is a unitary $U: M \rightarrow N$ such that $U A=B U$. In fact, this is just the unitary module isomorphism in the theory of Hilbert modules $[\mathbf{9}]$.

TheOREM 3.18. Suppose that $M($ resp. $N)$ is a stable graded $\mathcal{S}$-module, and the $\mathcal{S}$-calculus is given by $A$ (resp. B). If $M$ has a truncated stable decomposition as follows

$$
M=\bigoplus_{n=n_{1}}^{+\infty} M_{n}
$$

where $n_{1}=n(M)$, then $M$ and $N$ are $\mathcal{S}$-isomorphic if and only if $N$ has the truncated homogeneous decomposition as follows

$$
N=\bigoplus_{n=n_{2}}^{+\infty} N_{n}
$$

where $n_{2}=n(N)$, and for any $n \geq 0$ there exists a unitary operator $U_{n}: M_{n_{1}+n} \rightarrow N_{n_{2}+n}$ such that

$$
\left.B\right|_{N_{n_{2}+n}} U_{n}=\left.U_{n+1} A\right|_{M_{n_{1}+n}} .
$$

Proof. By the truncated stable decomposition given in the statement, it is clear that 


$$
M_{n_{1}+n}=\mathcal{S}^{n} M \ominus \mathcal{S}^{n+1} M, \quad n \in \mathbb{Z}_{+} .
$$

If $M$ and $N$ are $\mathcal{S}$-isomorphic, then there is a unitary $U: M \rightarrow N$ such that $U A=B U$. Then

$$
N=U M=\bigoplus_{n=n_{1}}^{+\infty} U M_{n}
$$

Since $N \ominus \mathcal{S}^{1} N=U\left(M \ominus \mathcal{S}^{1} M\right)=U M_{n_{1}} \neq 0$, by Proposition 3.12, the stable decomposition of $N$ must be truncated and uniquely determined. Suppose

$$
N=\bigoplus_{n=n_{2}}^{+\infty} N_{n}
$$

where $n_{2} \in \mathbb{Z}$. Then if $n \geq 0$,

$$
N_{n_{2}+n}=\mathcal{S}^{n} N \ominus \mathcal{S}^{n+1} N=U\left(\mathcal{S}^{n} M \ominus \mathcal{S}^{n+1} M\right)=U M_{n_{1}+n}
$$

holds. Taking $U_{n}=\left.U\right|_{M_{n_{1}+n}}$, since $U A=B U$ we get the require result.

Conversely, assume $M$ and $N$ have the truncated homogeneous decompositions as in the statement, and for any $n \in \mathbb{Z}_{+}$there exists a unitary $U_{n}$ from $M_{n_{1}+n}$ to $N_{n_{2}+n}$ such that $\left.B\right|_{N_{n_{2}+n}} U_{n}=\left.U_{n+1} A\right|_{M_{n_{1}+n}}$. Define a linear operator $U$ from $M$ to $N: U=\bigoplus U_{n}$, then $U$ is unitary. This gives a required $\mathcal{S}$-isomorphism between $M$ and $N$.

Hence, we find that under the stable condition the property of truncated is preserved by an $\mathcal{S}$-isomorphism. For the sake of brevity we can say that the unitary module isomorphism between truncated stable graded $\mathcal{S}$-modules is exactly the graded unitary module isomorphism.

Next, we apply this to the submodules of an $\mathcal{S}$-module. It is easy to see that $\mathcal{S}$-submodules and reducing subspaces are the same thing. Then we find that $\mathcal{S}$ isomorphism of submodules is exactly the unitary equivalence of reducing subspaces. Let $M$ and $N$ be two submodules of $\mathcal{S}$-module $H$, where $\mathcal{S}$-calculus is given by $T, M$ and $N$ are $\mathcal{S}$-isomorphic via $U$. We can define a bounded operator $V$ over $H$ as follows: $V x=U x$ if $x \in M, V x=0$ if $x \in M^{\perp}$. Then $V$ is a partial isometry in $\mathcal{V}^{*}(T)$ such that the initial space is $M$ and the final space is $N$. That is to say, the projections $P_{M}$ and $P_{N}$ are equivalent in $\mathcal{V}^{*}(T)$, where $P_{M}$ and $P_{N}$ have respective ranges $M$ and $N$. Now we state here a special case of Theorem 3.18.

Proposition 3.19. Suppose that $H$ is a graded $\mathcal{S}$-module, $M$ and $N$ are two stable graded submodules. If $M$ has a truncated stable decomposition as follows

$$
M=\bigoplus_{n=n_{1}}^{+\infty} M_{n}
$$

where $n_{1}=n(M)$, then $M$ and $N$ are $\mathcal{S}$-isomorphic if and only if $N$ has the truncated homogeneous decomposition as follows 


$$
N=\bigoplus_{n=n_{2}}^{+\infty} N_{n}
$$

where $n_{2}=n(N)$, and for any $n \geq 0$ there exists a unitary operator $U_{n}: M_{n_{1}+n} \rightarrow N_{n_{2}+n}$ such that

$$
\left.T\right|_{N_{n_{2}+n}} U_{n}=\left.U_{n+1} T\right|_{M_{n_{1}+n}}
$$

\section{Applications.}

We have developed the theory of graded structures for Hilbert spaces. As an application of these ideas, we will discuss the multiplication operators induced by some polynomials.

\subsection{The $\mathcal{V}^{*}\left(M_{z^{k}}\right)$ on $H^{2}(\omega)$.}

In this subsection, a complete description of $\mathcal{V}^{*}\left(M_{z^{k}}\right)$ on $H^{2}(\omega)$ is obtained. We mention that all reducing subspaces of $M_{z^{k}}$ was described in [31]. To state our main results, we need to introduce some notions and results that belong to Stessin and Zhu (see $[\mathbf{3 1}])$.

Fix a positive integer $k$, set $\Omega=\left\{n \in \mathbb{Z}_{+}: 0 \leq n \leq k-1\right\}$. We can introduce a equivalence relation $\sim$ on $\Omega$ as follows,

$$
a \sim b \Leftrightarrow \frac{\omega_{a+n k}}{\omega_{a}}=\frac{\omega_{b+n k}}{\omega_{b}}, \quad \forall n \in \mathbb{Z}_{+} .
$$

Suppose $p$ is a polynomial of degree less than $k$,

$$
p(z)=\sum_{n=0}^{k-1} a_{n} z^{n} .
$$

$p$ will be called transparent if for any two non-zero coefficients $a_{i}$ and $a_{j}$ we have $i \sim j$. It is clear that each monomial of degree less than $k$ is transparent. The following theorem that belongs to Stessin and Zhu [31] shows that the concept of transparent polynomial is related to the minimal reducing subspaces of $M_{z^{k}}$.

TheOREM 4.1 ([31]). Let $X$ be a reducing subspace of $M_{z^{k}}$ on $H_{\omega}^{2}$. Then $X$ is minimal if and only if there is a transparent polynomial $p$ such that

$$
X=\left[\left\{p z^{n k}: n \in \mathbb{Z}_{+}\right\}\right] .
$$

Of course, if $k=1$, then the only transparent polynomial is constant, thus we know that $M_{z}$ must be irreducible for any $\omega$.

Next, we will give two new results. Denote by $X_{p}$ the reducing subspace generated by $p$ for $M_{z^{k}}$. The first result is the characterization of unitary equivalence between minimal reducing subspaces. According to Theorem 4.1, we know that every minimal reducing subspace of $M_{z^{k}}$ must has the form $X_{p}$ for some transparent polynomial $p$. Now we can prove the following theorem about unitary equivalence. 
THEOREM 4.2. If $p$ and $q$ are two transparent polynomials, then $X_{p}$ and $X_{q}$ are unitary equivalent if and only if $p+q$ is also a transparent polynomial.

Proof. Suppose

$$
p(z)=\sum_{n=i}^{k-1} a_{n} z^{n}, \quad q(z)=\sum_{n=j}^{k-1} b_{n} z^{n},
$$

where $a_{i} \neq 0$ and $b_{j} \neq 0$. Then we have

$$
X_{p}=\mathbb{C}\{p\} \bigoplus \mathbb{C}\left\{p z^{k}\right\} \bigoplus \cdots, \quad X_{q}=\mathbb{C}\{q\} \bigoplus \mathbb{C}\left\{q z^{k}\right\} \bigoplus \cdots
$$

Applying $\mathcal{S}$-calculus for $M_{z^{k}}$, since $p$ and $q$ are both transparent polynomials, we can check that $X_{p}$ and $X_{q}$ are both graded $\mathcal{S}$-modules. Since $X_{p}$ and $X_{q}$ are both minimal, both of them are stable by Proposition 3.16.

If $X_{p}$ and $X_{q}$ are unitarily equivalent, then according to Theorem 3.18, it follows that for each $n \in \mathbb{Z}_{+}$, there exists a unitary operator $U_{n}: \mathbb{C}\left\{p z^{n k}\right\} \rightarrow \mathbb{C}\left\{q z^{n k}\right\}$ such that $M_{z^{k}} U_{n}=U_{n+1} M_{z^{k}}$. Thus we obtain a non-zero constant $c$ such that

$$
U_{0} p=c q
$$

Then

$$
U_{0}\left(M_{z^{k}}^{*}\right)^{n} M_{z^{k}}^{n} p=U_{0} \frac{\omega_{i+n k}}{w_{i}} p=c \frac{\omega_{i+n k}}{\omega_{i}} q, \quad n \in \mathbb{Z}_{+} .
$$

Also

$$
\begin{aligned}
& U_{0}\left(M_{z^{k}}^{*}\right)^{n} M_{z^{k}}^{n} p=\left(M_{z^{k}}^{*}\right)^{n} U_{n} M_{z^{k}}^{n} p=\left(M_{z^{k}}^{*}\right)^{n} M_{z^{k}}^{n} U_{0} p=c\left(M_{z^{k}}^{*}\right)^{n} M_{z^{k}}^{n} q=c \frac{\omega_{j+n k}}{\omega_{j}} q, \\
& n \in \mathbb{Z}_{+} .
\end{aligned}
$$

It follows that $i \sim j$, this shows that $p+q$ is also transparent.

Conversely, if $p+q$ is transparent, then $i \sim j$. For each $n \in \mathbb{Z}_{+}$we can define a unitary $U_{n}: \mathbb{C}\left\{p z^{n k}\right\} \rightarrow \mathbb{C}\left\{q z^{n k}\right\}$ as follows

$$
U_{n} p z^{n k}=c_{n} q z^{n k}, \quad n \in \mathbb{Z}_{+} .
$$

We can assume that all $c_{n}$ are positive. Since $\left\|p z^{n k}\right\|=c_{n}\left\|q z^{n k}\right\|$, a calculation using the condition $i \sim j$ shows that

$$
c_{n}^{2}=\frac{\sum_{s=i}^{k-1}\left|a_{s}\right|^{2} \omega_{s}}{\sum_{s=j}^{k-1}\left|b_{s}\right|^{2} \omega_{s}} .
$$

We find that all $c_{n}$ are a same constant $c$. Then

$$
U_{n+1} M_{z^{k}} p z^{n k}=U_{n+1} p z^{(n+1) k}=c q z^{(n+1) k}=M_{z^{k}} U_{n} p z^{n k}, \quad n \in \mathbb{Z}_{+},
$$

Thus $M_{z^{k}} U_{n}=U_{n+1} M_{z^{k}}$. This implies that $X_{p}$ and $X_{q}$ are unitarily equivalent by 
Theorem 3.18 again.

In order to obtain the second result about $\mathcal{V}^{*}\left(M_{z^{k}}\right)$, we need the following key proposition.

Proposition 4.3. $\quad \mathcal{V}^{*}\left(M_{z^{k}}\right)$ is $*$-isomorphic to a subalgebra of $M_{k}(\mathbb{C})$.

Proof. Let $H_{k}=\operatorname{span}\left\{z^{i}: i \in \Omega\right\}$. Then $H_{k}$ is of dimension $k$, and $\mathcal{B}\left(H_{k}\right) \cong$ $M_{k}(\mathbb{C})$. Let $P$ be a projection from $H^{2}(\omega)$ onto $H_{k}$. We define a liner map $\Phi$ from $\mathcal{V}^{*}\left(M_{z^{k}}\right)$ to $\mathcal{B}\left(H_{k}\right)$ as follows

$$
\begin{aligned}
\Phi: \mathcal{V}^{*}\left(M_{z^{k}}\right) & \rightarrow \mathcal{B}\left(H_{k}\right) \\
T & \mapsto P T P .
\end{aligned}
$$

In fact, we can claim that $P \in \mathcal{V}^{*}\left(M_{z^{k}}\right)^{\prime}$. For any $f \in H^{2}(\omega)$, we can write $f$ as following form

$$
f(z)=p_{0}(z)+z^{k} p_{1}(z)+z^{2 k} p_{2}(z)+\cdots,
$$

where $p_{i} \in H_{k}$ for all $i$. Then $P f=p_{0}$. For every $T \in \mathcal{V}^{*}\left(M_{z^{k}}\right)$, we have $T P f=T p_{0}$. Then

$$
P T f=P T\left(p_{0}+M_{z^{k}} p_{1}+M_{z^{k}}^{2} p_{2}+\cdots\right)=P\left(T p_{0}+M_{z^{k}} T p_{1}+M_{z^{k}}^{2} T p_{2}+\cdots\right)=P T p_{0} .
$$

Thus $P T=P T P$. Since $T$ is arbitrary, we obtain $P T^{*}=P T^{*} P$. It follows that

$$
P T=\left(P T^{*} P\right)^{*}=\left(P T^{*}\right)^{*}=T P .
$$

Hence we have $P \in \mathcal{V}^{*}\left(M_{z^{k}}\right)^{\prime}$.

This shows that $\Phi$ is a well defined $*$-algebraic homomorphism. If $\Phi(T)=0$, then by $T \in \mathcal{V}^{*}\left(M_{z^{k}}\right)$, it is easy to see that $T f=0$ by $f^{\prime}$ 's form. Thus $\Phi$ is injective. This implies the desired result.

Thus, $\mathcal{V}^{*}\left(M_{z^{k}}\right)$ is of finite dimension, and is isomorphic to a direct sum of a finite collect of matrix algebras. If we use the equivalence relation $\sim$ to parition the set $\Omega$ into equivalence classes $\Omega_{1}, \cdots, \Omega_{K}$ and let $n_{i}=\left|\Omega_{i}\right|, 1 \leq i \leq K$, then we can prove the following theorem.

THEOREM 4.4 .

$$
\mathcal{V}^{*}\left(M_{z^{k}}\right) \cong \bigoplus_{i=1}^{K} M_{n_{i}}(\mathbb{C}) .
$$

Proof. By the theory of von Neumann algebra [24], we can write

$$
\mathcal{V}^{*}\left(M_{z^{k}}\right) \cong \bigoplus_{i=1}^{n} M_{m_{i}}(\mathbb{C})
$$


We already see that every minimal reducing subspaces of $M_{z^{k}}$ has the form $X_{p}$ where $p$ is a transparent polynomial, and for any two transparent polynomial $p$ and $q, X_{p}$ and $X_{q}$ are unitary equivalent if and only if $p+q$ is transparent. This defines a equivalence relation on the set of transparent polynomials. The equivalence relation partitions them into equivalence classes $\Delta_{1}, \cdots, \Delta_{K}$. On the other hand, $\bigoplus_{i=1}^{n} M_{m_{i}}(\mathbb{C})$ implies that $n$ must be $K$, since the unitary equivalence relation on the set of minimal reducing subspaces is equivalent to the equivalence relation on the set of transparent polynomials, hence the respective numbers of equivalence classes must be same.

By interchange of indices, we can assume that for each $a \in \Omega$, when $a \in \Omega_{i}$ we have $X_{z^{a}} \in \Delta_{i}$ and $P_{X_{z^{a}}} \in M_{m_{i}}(\mathbb{C})$. Then for each $1 \leq i \leq K, \Delta_{i}$ has $n_{i}$ minimal reducing subspaces that have the form $X_{z^{a}}$ and are orthogonal to each other. Hence we find that $n_{i} \leq m_{i}$. In fact, it is easy to see that any minimal reducing subspace $X_{p}$ can be contained in the direct sum of $X_{z^{l_{j}}}$ where all $l_{j}$ belong to some $\Omega_{i}$. It forces that $n_{i}=m_{i}$. This completes the proof.

\subsection{The multiplication operator induced by a quasi-homogeneous poly- nomial.}

Our second application of the theory of graded structures will be to a multiplication operator induced by a quasi-homogeneous polynomial. Given an integer $d \geq 2$ and a weight

$$
K=\left(K_{1}, K_{2}, \cdots, K_{d}\right) \in \mathbb{N}^{d},
$$

we define the $K$-degree for every monomial $z^{\alpha} \in \mathbb{C}\left[z_{1}, \cdots, z_{d}\right]$ by $\sum_{i=1}^{d} \alpha_{i} K_{i}$. A polynomial $p \in \mathbb{C}\left[z_{1}, \cdots, z_{d}\right]$ is said to be $K$-quasi-homogeneous if its all monomials have the same $K$-degree. We further define the $K$-degree of zero polynomial is $-\infty$. If $K=(1,1, \cdots, 1)$, then $K$-quasi-homogeneous is homogeneous.

Fixed a weight $K$. Let $H$ be the Hilbert space consisting of analytic functions in a Reinhardt domain in $\mathbb{C}^{d}$ such that all monomials consist of a complete orthogonal set of $H$, and every multiplication operator induced by a polynomial is bounded. Then $H$ has the following form

$$
H=\bigoplus_{n=0}^{\infty} H_{n},
$$

where $H_{n}$ is the set consisting of all $K$-quasi-homogeneous polynomials of $K$-degree $n$. Suppose that $p$ is a $K$-quasi-homogeneous polynomial of $K$-degree $k$, we consider the multiplication operator $M_{p}$ on $H$. Let $q$ be a $K$-quasi-homogeneous polynomial of $K$-degree $l$. Applying $\mathcal{S}$-calculus for multiplication operator $M_{p}$, we can check that $\mathcal{S}^{n} q \subseteq H_{n k+l}$. We write $l=a k+b, 0 \leq b<k$, then the reducing subspace $[q]_{M_{p}}$ of $M_{p}$ generated by $q$ is a graded $\mathcal{S}$-module, and it has the homogeneous decomposition as follows

$$
[q]_{M_{p}}=\bigoplus_{n=-a}^{\infty} \mathcal{S}^{n} q .
$$


We have the following theorem.

THEOREM 4.5. $\quad[1]_{M_{p}}$ must be a minimal reducing subspace of $M_{p}$.

Proof. In this case, $l=0, a=0, b=0$, thus

$$
[1]_{M_{p}}=\bigoplus_{n=0}^{\infty} \mathcal{S}^{n} 1 .
$$

Note that for each integer $n \geq 0, \mathcal{S}^{1}\left(\mathcal{S}^{n} 1\right)=\mathcal{S}^{n+1} 1$. Since $\mathcal{S}^{0} 1=\mathbb{C}$, it is true that $\operatorname{dim} \mathcal{S}^{0} 1=1$. By Proposition 3.17, the desired result is obtained.

Thus, we quickly find that a multiplication operator induced by a quasi-homogeneous polynomial must has a minimal reducing subspace generated by 1 .

\section{3. $M_{z+w}$ on $H^{2}(\omega, \delta)$.}

In the preceding subsections, we discussed monomials and quasi-homogeneous polynomials. Now we will learn more on $M_{z+w}$ on $H^{2}(\omega, \delta)$. Applying $\mathcal{S}$-calculus for the multiplication operator $M_{z+w}$ and writing $H_{r}=\left[\left\{z^{k} w^{l}: k+l=r\right\}\right]$ for each $r \in \mathbb{Z}_{+}$, we have $H^{2}(\omega, \delta)=\bigoplus_{r=0}^{\infty} H_{r}$ and $\mathcal{S}^{n} H_{r} \subseteq H_{n+r}$. Then $H^{2}(\omega, \delta)$ is a graded $\mathcal{S}$-module, and Theorem 4.5 reveals that $[1]_{\mathcal{S}}$ is a minimal reducing subspace. In this section, we mainly give a concise summary of the results in $[\mathbf{1 2}],[\mathbf{2 5}]$. There are two primary questions:

Question 1: When does $[1]_{\mathcal{S}}=H^{2}(\omega, \delta)$ hold?

Question 2: Given another two sequences of positive numbers $\omega^{\prime}$ and $\delta^{\prime}$, when are $M_{z+w}$ on $H^{2}(\omega, \delta)$ and $M_{z+w}$ on $H^{2}\left(\omega^{\prime}, \delta^{\prime}\right)$ unitarily equivalent?

Note that $H^{2}(\omega, \delta)=H^{2}(\omega) \otimes H^{2}(\delta)$ and $M_{z+w}=M_{z} \otimes I+I \otimes M_{w}$, where $M_{z}$ (resp. $M_{w}$ ) is a bounded operator on $H^{2}(\omega)$ (resp. $H^{2}(\delta)$ ). In general, suppose that $H$ and $K$ are two Hilbert spaces. If $A \in B(H)$ and $B \in B(K)$, then $M=A \otimes I+I \otimes B$ is a bounded operator on the Hilbert space $H \otimes K$. The two questions above then are generalized to the following:

Question 3: If both $A$ and $B$ are irreducible, when is $M$ irreducible?

Question 4: Given $A^{\prime} \in B(H), B^{\prime} \in B(K)$, and $M^{\prime}=A^{\prime} \otimes I+I \otimes B^{\prime}$, when are $M$ and $M^{\prime}$ unitarily equivalent?

For Question 3, the following proposition may be useful.

Proposition $4.6([\mathbf{2 5}])$. Suppose that $H$ and $K$ are both of dimensions at least two. Let $A \in B(H)$ and $B \in B(K)$, if $A$ and $B$ are unitarily equivalent, then $A \otimes I+I \otimes B$ is reducible.

Remark 4.7. However, even if $A$ and $B$ are not unitarily equivalent, $A \otimes I+I \otimes B$ may be reducible. A simple example is given by putting $H=K=\mathbb{C}^{2}, A=\left(\begin{array}{ll}1 & 1 \\ 0 & 1\end{array}\right)$, $B=\left(\begin{array}{ll}0 & 1 \\ 0 & 0\end{array}\right)$, and $M=A \otimes I+I \otimes B$. Then both $A$ and $B$ are irreducible and not unitarily equivalent, while the one-dimensional subspace spanned by $\left(\begin{array}{l}1 \\ 0\end{array}\right) \otimes\left(\begin{array}{l}0 \\ 1\end{array}\right)-\left(\begin{array}{l}0 \\ 1\end{array}\right) \otimes\left(\begin{array}{l}1 \\ 0\end{array}\right)$ is reducing for $M$. The authors thank the referee for providing this example. In fact, we can write $M=I \otimes I+I \otimes B+B \otimes I$ and quickly find that $M$ is reducible by Porposition 4.6. 
It is well known that $M_{z}$ on $H^{2}(\omega)$ and $M_{w}$ on $H^{2}(\delta)$ are unitarily equivalent if and only if $\left(\omega_{n+1}\right) / \omega_{n}=\left(\delta_{n+1}\right) / \delta_{n}$ for all $n \in \mathbb{Z}_{+}$. In this case, we write $\omega \sim \delta$. Thus, if $\omega \sim \delta$, then the answer to the Question 1 is NO. Based on this, we proposed the following conjecture.

Conjecture 4.8. $M_{z+w}$ on $H^{2}(\omega, \delta)$ is reducible only if $\omega \sim \delta$.

In [25], we proved that there is a class $\mathcal{F}$ of unilateral weighted shifts such that if $A$ and $B$ are both in $\mathcal{F}$, then $A \otimes I+I \otimes B$ is irreducible if and only if $A$ and $B$ are not unitarily equivalent. Can $\mathcal{F}$ be the whole class of unilateral weighted shifts, even a larger class? The conjecture and the Question 2 are of course very complicated, we have to do more research on these in future.

\section{4. $M_{z}+M_{w}^{*}$ on $H^{2}\left(\mathbb{D}^{2}\right)$.}

In the previous sections, the examples we investigated are all truncated. Next we will study a non-truncated example that already arose in Example 3.9. We will find that the result provides a nontrivial example to the Question 3 that proposed in Subsection 4.3. That is, if $A$ and $B$ are two irreducible operators and not unitarily equivalent, then is the operator $A \otimes I+I \otimes B$ irreducible?

Let $A=M_{z}$ be the unilateral shift on $H^{2}(\mathbb{D})$ and $B=M_{w}^{*}$ be the unilateral backward shift on $H^{2}(\mathbb{D})$. It is easy to see that $A$ and $B$ are not unitarily equivalent and that $A$ and $B$ are irreducible. Specifically, the base space is $H^{2}(\mathbb{D}) \otimes H^{2}(\mathbb{D})=H^{2}\left(\mathbb{D}^{2}\right)$, the Hardy space over the bidisk, and the operator that we concern is $M_{z}+M_{w}^{*}$, which is exactly the Toeplitz operator with the symbol $z+\bar{w}$ on $H^{2}\left(\mathbb{D}^{2}\right)$.

Set $M=M_{z}+M_{w}^{*}$ and the commutator $C=\left[M^{*}, M\right]=M^{*} M-M M^{*}$. It is easy to see that $C=\left[M_{z}^{*}, M_{z}\right]+\left[M_{w}, M_{w}^{*}\right]$. Then we have

$$
C z^{n} w^{m}=\left\{\begin{array}{l}
0, \quad n=m=0 \text { or } n m \neq 0 \\
w^{m}, \quad n=0, m \neq 0 \\
-z^{n}, n \neq 0, m=0
\end{array}\right.
$$

Take $H_{n}=\left[\left\{z^{i} w^{j}: i-j=n\right\}\right]$. Then

$$
H^{2}\left(\mathbb{D}^{2}\right)=\bigoplus_{n=-\infty}^{\infty} H_{n}
$$

Applying $\mathcal{S}$-calculus for $M$, we have

$$
\mathcal{S}^{n} H_{m} \subseteq H_{n+m}, \quad n, m \in \mathbb{Z} .
$$

Hence $H^{2}\left(\mathbb{D}^{2}\right)$ is turned into a graded $\mathcal{S}$-module that is non-truncated.

Next, we check that whether $H^{2}\left(\mathbb{D}^{2}\right)$ is stable. We begin with the following lemma.

LEMMA 4.9. If $n \neq-1$, then $\mathcal{S}^{1} H_{n}=H_{n+1}$.

Proof. If $n \geq 0, H_{n}=\left[\left\{z^{n+k} w^{k}: k \in \mathbb{Z}_{+}\right\}\right]$. If $k=0$, we have $z^{n+1}=M z^{n} \in$ $\mathcal{S}^{1} H_{n}$. Suppose that a non-negative integer $k$ such that $z^{n+k+1} w^{k} \in \mathcal{S}^{1} H_{n}$. Then 


$$
M z^{n+k+1} w^{k+1}=z^{n+k+2} w^{k+1}+z^{n+k+1} w^{k} \in \mathcal{S}^{1} H_{n} .
$$

It follows that $z^{n+k+2} w^{k+1} \in \mathcal{S}^{1} H_{n}$. By induction, we get $\mathcal{S}^{1} H_{n}=H_{n+1}$.

If $n<-1, H_{n}=\left[\left\{z^{k} w^{k-n}: k \in \mathbb{Z}_{+}\right\}\right]$. If $k=0$, we have $M w^{-n}=z w^{-n}+w^{-n-1}$. Since $n<-1$, we find that $w^{-n-1}=C M w^{-n} \in \mathcal{S}^{1} H_{n}$. Hence, $z w^{-n} \in \mathcal{S}^{1} H_{n}$. If $k>0$, the similar argument as above will show that $\mathcal{S}^{1} H_{n}=H_{n+1}$.

Take $E_{0}=\left[\left\{z^{k} w^{k}(1+z w): k \in \mathbb{Z}_{+}\right\}\right]$. Then we have the following lemma.

LEMMA 4.10. $H_{0}=E_{0}$.

Proof. It is clearly that $H_{0} \supseteq E_{0}$. Suppose that a function $f(z, w) \in H_{0}$ such that $f \perp E_{0}$. Write $f=\sum_{k} a_{k} z^{k} w^{k}$. Since $f \perp z^{k} w^{k}(1+z w)$, it follows that $a_{k}+a_{k+1}=0$. We conclude that $f=a_{0}\left(1-z w+z^{2} w^{2}+\cdots\right)$. As $f \in H^{2}\left(\mathbb{D}^{2}\right), f$ must be zero. This implies that $H_{0}=E_{0}$.

We are now in a position to prove that $H^{2}\left(\mathbb{D}^{2}\right)$ is stable.

LEMMA 4.11. $\mathcal{S}^{1} H_{-1}=H_{0}$.

Proof. $\quad H_{-1}=\left[\left\{z^{k} w^{k+1}: k \in \mathbb{Z}_{+}\right\}\right]$. For each $k \in \mathbb{Z}_{+}$, we have $M z^{k} w^{k+1}=$ $z^{k+1} w^{k+1}+z^{k} w^{k}=z^{k} w^{k}(1+z w)$. Thus, we conclude that $H_{0} \supseteq \mathcal{S}^{1} H_{-1} \supseteq E_{0}=H_{0}$, as required. The last equality holds because of Lemma 4.10

Using similar argument as above, we can prove the following lemma.

Lemma 4.12. $\mathcal{S}^{-1} H_{n}=H_{n-1}$, for every integer $n$.

Next, we will show that $H^{2}\left(\mathbb{D}^{2}\right)$ is surely minimal. The following lemma establishes the first step.

LEMMA 4.13. If $k, l \in \mathbb{Z}_{+}$and $k \neq l$, then $\left[z^{k} w^{l}\right]_{\mathcal{S}}=H^{2}\left(\mathbb{D}^{2}\right)$.

Proof. To begin, we verify that $\mathcal{S}^{0}\left\{z^{k} w^{l}\right\}=H_{k-l}$. If $k>l>0$, then

$$
\begin{aligned}
C M^{* l} C M^{l} z^{k} w^{l} & =C M^{* l} C\left(z^{k}+l z^{k+1} w+\cdots+z^{k+l} w^{l}\right) \\
& =-C M^{* l} z^{k} \\
& =-C\left(z^{k-l}+l z^{k-l+1} w+\cdots+z^{k} w^{l}\right) \\
& =z^{k-l} .
\end{aligned}
$$

It follows that $z^{k-l} \in \mathcal{S}^{0}\left\{z^{k} w^{l}\right\}$. This result is also true if $l=0$. If $0<k<l$, then

$$
\begin{aligned}
C M^{k} C M^{* k} z^{k} w^{l} & =C M^{k} C\left(w^{l}+k z w^{l+1}+\cdots+z^{k} w^{l+k}\right) \\
& =C M^{k} w^{l} \\
& =C\left(w^{l-k}+k z w^{l-k+1}+\cdots+z^{k} w^{l}\right) \\
& =w^{l-k} .
\end{aligned}
$$


It follows that $w^{l-k} \in \mathcal{S}^{0}\left\{z^{k} w^{l}\right\}$. This result is also true if $k=0$.

If $k>l$, we have shown that $z^{k-l} \in \mathcal{S}^{0}\left\{z^{k} w^{l}\right\}$. Then

$$
M^{*} M z^{k-l}=M^{*} z^{k-l+1}=z^{k-l}+z^{k-l+1} w,
$$

thus $z^{k-l+1} w \in \mathcal{S}^{0}\left\{z^{k} w^{l}\right\}$. Suppose that an integer $n \geq 1$ such that for all $0 \leq m \leq n$, $z^{k-l+m} w^{m} \in \mathcal{S}^{0}\left\{z^{k} w^{l}\right\}$. Then

$$
\begin{aligned}
M^{*} M z^{k-l+n} w^{n} & =M^{*}\left(z^{k-l+n+1} w^{n}+z^{k-l+n} w^{n-1}\right) \\
& =z^{k-l+n-1} w^{n-1}+2 z^{k-l+n} w^{n}+z^{k-l+n+1} w^{n+1} .
\end{aligned}
$$

It follows that $z^{k-l+n+1} w^{n+1} \in \mathcal{S}^{0}\left\{z^{k} w^{l}\right\}$. Thus $\mathcal{S}^{0}\left\{z^{k} w^{l}\right\}=H_{k-l}$ by induction. The argument for $k<l$ is similar.

We have established that $\mathcal{S}^{0}\left\{z^{k} w^{l}\right\}=H_{k-l}$. By Lemma 4.9, Lemma 4.11 and Lemma 4.12, we have $\mathcal{S}^{n}\left\{z^{k} w^{l}\right\}=\mathcal{S}^{n} H_{k-l}=H_{k-l+n}$ for all integer $n$. It follows from Proposition 3.10 that $\left[z^{k} w^{l}\right]_{\mathcal{S}}=H^{2}\left(\mathbb{D}^{2}\right)$.

Finally, we need a definition. The order of an analytic function $f(z, w)=$ $\sum_{k, l} a_{k l} z^{k} w^{l}$ with respect to $w$, is by definition

$$
\operatorname{ord}_{w}(f)=\min \left\{l: a_{k l} \neq 0\right\} .
$$

Now, we can prove that $M=M_{z}+M_{w}^{*}$ is irreducible. The reader is easy to verify ker $M=0$. In what following will need this fact.

TheOREM 4.14. $H^{2}\left(\mathbb{D}^{2}\right)$ is minimal, that is, $H^{2}\left(\mathbb{D}^{2}\right)$ is the exactly unique, not vanishing reducing subspace of $M_{z}+M_{w}^{*}$.

Proof. Let $\varphi \in H^{2}\left(\mathbb{D}^{2}\right)$ and $\varphi \neq 0$. We must prove that $[\varphi]_{\mathcal{S}}=H^{2}\left(\mathbb{D}^{2}\right)$. Suppose that $\varphi(z, w)=\sum_{k, l} a_{k l} z^{k} w^{l}$. If $\varphi \in H_{0}$, we can replace $\varphi$ by $M \varphi$ and $M \varphi \in[\varphi]_{\mathcal{S}}$ because $M \varphi \neq 0$. Hence, we can assume that $\varphi \notin H_{0}$. Then there exist integers $i, j, r$ such that $i-j=r \neq 0$ and $a_{i j} \neq 0$. Write $\varphi(z, w)$ as $\varphi(z, w)=s(z, w)+t(z, w)$ such that $s \in H_{r}$ and $t \perp H_{r}$. Notice that $s \neq 0$. For simplicity, we may take $j=\operatorname{ord}_{w} s$. In this case, it is easy to see that $i=\operatorname{ord}_{z} s$. Take $s_{1}(z, w)=s(z, w)-a_{i j} z^{i} w^{j}$, then we have

$$
\begin{aligned}
C M^{j} \varphi(z, w) & =C M^{j} a_{i j} z^{i} w^{j}+C M^{j} s_{1}(z, w)+C M^{j} t(z, w) \\
& =-a_{i j} z^{i}+C M^{j} t(z, w) .
\end{aligned}
$$

Since $C M^{j} t \perp z^{i}, C M^{j} \varphi(z, w) \neq 0$, and hence we can replace $\varphi$ by $C M^{j} \varphi(z, w)$. Therefore we may assume that $\varphi$ is such that there is an integer $i>0$ satisfying $a_{i 0} \neq 0$.

Take $f(z)=\sum_{k>0} a_{k 0} z^{k}, g(w)=\sum_{l>0} a_{0 l} w^{l}$, and $h(z, w)=\sum_{k>0, l>0} a_{k l} z^{k-1} w^{l-1}$. Then we have

$$
\varphi(z, w)=a_{00}+f(z)+g(w)+z w h(z, w),
$$

where $f \neq 0$. It is easy to see that $C \varphi(z, w)=-f(z)+g(w)$. Thus 


$$
a_{00}+2 f(z)+z w h(z, w)=\varphi(z, w)-C \varphi(z, w) \in \mathcal{S}^{0}\{\varphi\} .
$$

Applying $C$ again for the above, we get that $f(z) \in \mathcal{S}^{0}\{\varphi\}$.

Since $f(0)=0$, there is a positive integer $n$ such that $f(z)=z^{n} f_{0}(z)$ and $f_{0}(0) \neq 0$. We will now prove by induction that for all $n \in \mathbb{N},\left[z^{n} f_{0}(z)\right]_{\mathcal{S}}=H^{2}\left(\mathbb{D}^{2}\right)$. If $n=1$, then

$$
M C M^{*} z f_{0}(z)=M C\left(f_{0}(z)+w z f_{0}(z)\right)=M\left(f_{0}(0)-f_{0}(z)\right)=f_{0}(0) z-z f_{0}(z) .
$$

Thus, $f_{0}(0) z=z f_{0}(z)+M C M^{*} z f_{0}(z)$. Consequently, $z \in\left[z f_{0}(z)\right]_{\mathcal{S}}$. By Lemma 4.13 , we conclude that $\left[z f_{0}(z)\right]_{\mathcal{S}}=[z]_{\mathcal{S}}=H^{2}\left(\mathbb{D}^{2}\right)$. Next, assume that $\left[z^{n} f_{0}(z)\right]_{\mathcal{S}}=H^{2}\left(\mathbb{D}^{2}\right)$ for $n$. Then

$$
C M^{*} z^{n+1} f_{0}(z)=C\left(z^{n} f_{0}(z)+w z^{n+1} f_{0}(z)\right)=-z^{n} f_{0}(z) .
$$

Thus by the induction hypothesis, $\left[z^{n+1} f_{0}(z)\right]_{\mathcal{S}}=\left[z^{n} f_{0}(z)\right]_{\mathcal{S}}=H^{2}\left(\mathbb{D}^{2}\right)$, as required. We have now established that $[\varphi]_{\mathcal{S}}=\left[z^{n} f_{0}(z)\right]_{\mathcal{S}}=H^{2}\left(\mathbb{D}^{2}\right)$.

Just recently, Deng, Lu and Shi completely characterized all reducing subspaces for $\alpha M_{z}^{k}+\beta M_{w}^{* l}$ on the Bergman space over bidisk [13]. Their work also shows that $M_{z}+M_{w}^{*}$ is irreducible in the case of Bergman space.

ACKnowledgements. The authors thank the editor and the referee for providing constructive comments and help in improving this paper.

\section{References}

[ 1 ] W. Arveson, The curvature of a Hilbert module over $\mathbb{C}\left[z_{1}, \ldots, z_{d}\right]$, Proc. Natl. Acad. Sci. USA, 96 (1999), 11096-11099.

[2] W. Arveson, The curvature invariant of a Hilbert module over $\mathbb{C}\left[z_{1}, \ldots, z_{d}\right]$, J. Reine Angew. Math., 522 (2000), 173-236.

[3] R. Bhatia and P. Rosenthal, How and why to solve the operator equation $A X-X B=Y$, Bull. London Math. Soc., 29 (1997), 1-21.

[ 4 ] L. Brown, R. Douglas and P. Fillmore, Unitary equivalence modulo the compact operators and extension of $C^{*}$-algebras, Proceedings of Conference on Operator Theory (Dalhousie Univ., Halifax, N. S.), Lecture Notes in Math., 345, Springer, Berlin, 1973, 58-128.

[5] J. Carlson, D. Clark, C. Foias and J. Williams, Projective Hilbert $A(\mathbb{D})$-modules, New York J. Math., 1 (1994), 26-38.

[6] J. Carlson and D. Clark, Cohomology and extensions of Hilbert modules, J. Funct. Anal., 128 (1995), 278-306.

[ 7 ] J. Carlson and D. Clark, Projectivity and extensions of Hilbert modules over $A\left(\mathbb{D}^{N}\right)$, Mich. Math. J., 44 (1997), 365-373.

[ 8 ] X. Chen and R. Douglas, Localization of Hilbert modules, Mich. Math. J., 39 (1992), $443-454$.

[ 9 ] X. Chen and K. Guo, Analytic Hilbert Modules, Research Notes in Mathematics, 433, Chapman \& $\mathrm{Hal} / \mathrm{CRC}, 2003$.

[10] J. Conway, A Course in Operator Theory, Graduate Studies in Mathematics, 21, AMS, Rhode Island, 2000.

[11] M. Cowen and R. Douglas, Complex geometry and operator theory, Acta Math., 141 (1978), 187-261.

[12] H. Dan and H. Huang, Multiplication operators defined by a class of polynomials on $L_{a}^{2}\left(\mathbb{D}^{2}\right)$, Integr. Equ. Oper. Theory, 80 (2014), 581-601.

[13] J. Deng, Y. Lu and Y. Shi, Reducing subspaces for a class of non-analytic Toeplitz operators on the bidisk, J. Math. Anal. Appl., 445 (2017), 784-796. 
[14] R. Douglas and G. Misra, Equivalence of quotient Hilbert modules, Proc. Indian Acad. Sci. (Math. Sci.), 113 (2003), 281-291.

[15] R. Douglas and G. Misra, Equivalence of quotient Hilbert modules II, Trans. Am. Math. Soc., 360 (2008), 2229-2264.

[16] R. Douglas and V. Paulsen, Hilbert modules over function algebras, Pitman Research Notes in Mathematics Series, 217, Longman Scientific \& Technical, Harlow, 1989.

[17] R. Douglas, V. Paulsen and K. Yan, Operator theory and algebraic geometry, Bull. Amer. Math. Soc., 20 (1989), 67-71.

[18] R. Douglas and K. Yan, Hilbert-Samuel polynomials for Hilbert modules, Indiana Univ. Math. J., 42 (1993), 811-820.

[19] Y. Duan and K. Guo, Dimension formula for localization of Hilbert modules, J. Oper. Theory, 62 (2009), 439-452.

[20] D. Eisenbud, Commutative Algebra with a View Toward Algebraic Geometry, Graduate Texts in Mathematics, 150, Springer, 2004.

[21] X. Fang, Samuel multiplicity and the structure of semi-Fredholm operators, Adv. Math., 186 (2004), 411-437.

[22] X. Fang, The Fredholm index of quotient Hilbert modules, Math. Res. Lett., 12 (2005), 911-920.

[23] K. Guo, Characteristic spaces and rigidity for analytic Hilbert modules, J. Funct. Anal., 163 (1999), 133-151.

[24] K. Guo and H. Huang, Multiplication Operators on the Bergman Space, Lecture Notes in Math., 2145, Springer, 2015.

[25] K. Guo and X. Wang, Reducing subspaces of tensor products of weighted shifts, Sci. China Math., 59 (2016), 715-730.

[26] Y. Lu and X. Zhou, Invariant subspaces and reducing subspaces of weighted Bergman space over bidisk, J. Math. Soc. Japan, 62 (2010), 745-765.

[27] J. Sarkar, An introduction to Hilbert module approach to multivariable operator theory, Operator Theory, Springer, 2015, 969-1033.

[28] J. Sarkar, Applications of Hilbert module approach to multivariable operator theory, Operator Theory, Springer, 2015, 1035-1091.

[29] Y. Shi and Y. Lu, Reducing subspaces for Toeplitz operators on the polydisk, Bull. Korean Math. Soc., 50 (2013), 687-696.

[30] A. Shields, Weighted shift operators and analytic function theory, Topics in operator theory, Math. Surveys, 13 (1974), 49-128.

[31] M. Stessin and K. Zhu, Reducing subspaces of weighted shift operators, Proc. Amer. Math. Soc., 130 (2002), 2631-2639.

[32] X. Wang, H. Dan and H. Huang, Reducing subspaces of multiplication operators with the symbol $\alpha z^{k}+\beta w^{l}$ on $L_{a}^{2}\left(\mathbb{D}^{2}\right)$, Sci. China Math., 58 (2015), 2167-2180.

\section{Kunyu GuO}

School of Mathematical Sciences

Fudan University

Shanghai 200433, China

E-mail: kyguo@fudan.edu.cn

\section{Xudi WANG}

Xi'an University of Technology

Xi'an 710048, China

E-mail: wangxd512@xaut.edu.cn 